\title{
COMPLETE ORTHONORMAL SEQUENCES OF FUNCTIONS UNIFORMLY SMALL ON A SUBSET
}

\section{J. A. HUMMEL ${ }^{1}$}

Given a region $G$ in the complex plane having a nonvoid interior and two complex valued functions, $f$ and $g$, defined in $G$, denote

$$
(f, g)_{G}=\iint_{G} f \bar{g} d x d y, \quad\|f\|_{G}=(f, f)_{G}^{1 / 2} .
$$

Let $D$ be a bounded plane domain and let $L^{2}(D)$ be the Hilbert space of all analytic functions in $D$ satisfying $\|f\|_{D}<\infty$. It can easily be shown that there exists a continuous, real valued function $m_{D}(z)$ in $D$ such that for any $f$ in $L^{2}(D)$ and any $z$ in $D$

$$
|f(z)| \leqq m_{D}(z)\|f\|_{D},
$$

(see, for example, $[1$, p. 5]).

Let $\phi_{n}$ be any complete orthonormal sequence in $L^{2}(D)$. Then from (1) follows

$$
\sum_{n=1}^{\infty}\left|\phi_{n}(z)\right|^{2} \leqq\left[m_{D}(z)\right]^{2},
$$

and hence the convergence of the expansion for the Bergman reproducing kernel

$$
K_{D}(z, w)=\sum_{n=1}^{\infty} \phi_{n}(z)\left[\phi_{n}(w)\right]-
$$

where $\left[\phi_{n}(w)\right]$ - indicates the complex conjugate of $\left[\phi_{n}(w)\right]$, (cf. $[1$, pp. 6 and 9$])$.

Let $K$ be a given compact subset of $D$. Then we might wish to try to approximate the kernel function in $K$ by using a finite series. Because of (2) and the fact that $m_{D}(z)$ is continuous, it is clear that given an $\epsilon>0$ and a complete orthonormal sequence $\left\{\phi_{n}\right\}$, there exists an $N=N\left(D, K, \epsilon,\left\{\phi_{n}\right\}\right)$ such that $m \geqq N$ and $z, w \in K$ implies

$$
\left|K_{D}(z, w)-\sum_{n=1}^{m} \phi_{n}(z)\left[\phi_{n}(w)\right]^{-}\right|<\epsilon .
$$

It might be asked whether or not $N$ can be chosen independently of

Presented to the Society, August 24, 1956; received by the editors September 10, 1956.

1 The writer holds a National Science Foundation postdoctoral fellowship. 
$\left\{\phi_{n}\right\}$, i.e., if we can find an upper bound to the number of terms required in the series of (3) to approximate $K_{D}(z, w)$ in $K$, independently of which orthonormal sequence $\left\{\phi_{n}\right\}$ is used.

In this note, we show that such an upper bound cannot exist. To do this, we first prove a result which the writer and several of those he has shown it to consider quite remarkable. If $K$ is a compact subset of $D$, and $\epsilon>0$ is given, then there exists a complete orthonormal sequence $\left\{\phi_{n}\right\}$ in $L^{2}(D)$ such that for all $z \in K$ and all $n,\left|\phi_{n}(z)\right|<\epsilon$.

The method of proof used is of some interest since it is one of relatively few examples of the use of doubly orthogonal functions.

Theorem 1. Let $D$ be a bounded domain in the complex plane, $L^{2}(D)$ the Hilbert space of all analytic functions in $D$ for which $\|f\|_{D}<\infty$, and $K$ a compact subset of $D$. Let $\epsilon>0$ be given. Then there exists a complete orthonormal sequence $\left\{\phi_{n}(z)\right\}$ in $L^{2}(D)$ such that $\left\|\phi_{n}\right\|_{K}<\epsilon$ for all $n$.

Proof. We may assume that $K$ is the closure of a domain contained in $D$, for if not we merely enlarge $K$ to $K^{\prime}$ satisfying this property.

Under these hypotheses we have the existence of a doubly orthogonal sequence of functions $\left\{\psi_{n}(z)\right\}$ (cf. $[1, \mathrm{pp} .14-17]$ ), that is, a complete orthonormal sequence in $L^{2}(D)$ satisfying

$$
\left(\psi_{n}, \psi_{m}\right)_{K}=\lambda_{n} \delta_{n m}, \quad \lambda_{n} \searrow 0 .
$$

Indeed, $\sum \lambda_{n}<\infty$, but we do not need this here. Since $\lambda_{n} \rightarrow 0$, we have

$$
\frac{1}{m} \sum_{n=1}^{m} \lambda_{n} \rightarrow 0 \text { as } m \rightarrow \infty .
$$

Given $\epsilon>0$, choose $N$ such that

$$
\frac{1}{2^{N}} \sum_{n=1}^{2^{N}} \lambda_{n}<\epsilon^{2}, \quad \lambda_{n}<\epsilon^{2} \text { for } n>2^{N} .
$$

Let $E_{N}=\left(\epsilon_{i j}\right)$ be a $2^{N} \times 2^{N}$ matrix consisting entirely of elements $\epsilon_{i j}= \pm 1$ such that any two rows are orthogonal. The existence of such matrices is of course well known. One is easily constructed inductively, setting

$$
E_{1}=\left(\begin{array}{rr}
1 & 1 \\
1 & -1
\end{array}\right), \quad E_{n+1}=\left(\begin{array}{rr}
E_{n} & E_{n} \\
E_{n} & -E_{n}
\end{array}\right)
$$

Set 


$$
\begin{aligned}
\phi_{i} & =\left(1 / 2^{N}\right)^{1 / 2} \sum_{j=1}^{2^{N}} \epsilon_{i j} \psi_{j}, & i=1,2, \cdots, 2^{N} . \\
& =\psi_{i}, & i>2^{N} .
\end{aligned}
$$

This set of functions is clearly orthonormal and complete in $L^{2}(D)$. From (4) and (5) we see that the desired conclusion, $\left\|\phi_{n}\right\|_{K}<\epsilon$, holds for $n>2^{N}$, while if $n \leqq 2^{N}$, then

$$
\left\|\phi_{n}\right\|_{K}^{2}=\frac{1}{2^{N}} \sum_{j=1}^{2^{N}} \lambda_{j}<\epsilon^{2} .
$$

Corollary 1. If $K$ is a compact subset of the bounded plane domain $D$ and if $\epsilon>0$ is given, then there exists a complete orthonormal sequence $\left\{\phi_{n}(z)\right\}$ in $L^{2}(D)$ such that for all $z \in K$ and all $n$

$$
\left|\phi_{n}(z)\right|<\epsilon \text {. }
$$

Proof. Let $G$ be a domain containing $K$ and such that $\bar{G} \subset D$. Let (6)

$$
m=\max _{z \in K} m_{G}(z)
$$

where $m_{G}(z)$ is defined as in (1).

From Theorem 1, construct an orthonormal sequence $\left\{\phi_{n}\right\}$ in $L^{2}(D)$ such that

$$
\left\|\phi_{n}\right\|_{a}<\frac{\epsilon}{m}
$$

for all $n$. Then because of (1), for any $z \in K$ and any $n$

$$
\left|\phi_{n}(z)\right| \leqq m_{G}(z)\left\|\phi_{n}\right\|_{G}<\epsilon .
$$

Corollary 2. Let $K$ be a compact subset of the bounded plane domain $D$. Let $0<\epsilon<\max K_{D}(z, z) / 2$ for $z \in K$. Then there exists no integer $N$ such that

$$
\left|K_{D}(z, w)-\sum_{n=1}^{N} \phi_{n}(z)\left[\phi_{n}(w)\right]-\right|<\epsilon
$$

for all $z, w \in K$ and any complete orthonormal sequence $\left\{\phi_{n}\right\}$ in $L^{2}(D)$.

Proof. Let $N$ be given. Choose $z_{0}$ in $K$ such that $K_{D}\left(z_{0}, z_{0}\right)>2 \epsilon$. From Corollary 1 , choose a complete orthonormal sequence $\left\{\phi_{n}\right\}$ such that $\left|\phi_{n}\left(z_{0}\right)\right|^{2}<\epsilon / N$ for all $n$. But then

$$
\left|K_{D}\left(z_{0}, z_{0}\right)-\sum_{n=1}^{N} \phi_{n}\left(z_{0}\right)\left[\phi_{n}\left(z_{0}\right)\right]-\right| \geqq K_{D}\left(z_{0}, z_{0}\right)-\sum_{n=1}^{N}\left|\phi_{n}\left(z_{0}\right)\right|^{2}>\epsilon .
$$


Finally we note that Theorem 1 is not confined to the space $L^{2}(D)$. For the proof we require only the existence of a doubly orthogonal sequence $\psi_{n}$ with $\lambda_{n} \rightarrow 0$. Thus, we have the result:

Theorem 2. Let $H_{1}$ and $H_{2}$ be two complete, separable Hilbert spaces and let $J: H_{2} \rightarrow H_{1}$ be a linear mapping of $H_{2}$ into $H_{1}$. Suppose that $J$ is completely continuous. Then, given $\epsilon>0$, there exists in $H_{2}$ a complete orthonormal sequence $\left\{\phi_{n}\right\}$ such that $\left\|J \phi_{n}\right\|_{1}<\epsilon$ for all $n$.

Proof. It is easily proved that if $J$ is completely continuous, there exists a doubly orthogonal sequence $\left\{\psi_{n}\right\}$, complete in $H_{2}$, and satisfying

$$
\begin{array}{rlrl}
\left(\psi_{n}, \psi_{m}\right)_{2} & =\delta_{n m}, \\
\left(J \psi_{n}, J \psi_{m}\right)_{1} & =\lambda_{n} \delta_{n m}, & & \lambda_{n} \searrow 0 .
\end{array}
$$

The proof of this theorem then proceeds exactly as that of Theorem 1 .

\section{BIBLIOGRAPHY}

1. S. Bergman, The kernel function and conformal mapping, Math. Surveys, No. 5, American Mathematical Society, New York, 1950.

STANFORD UNIVERSITY 